\title{
Editorial: Indicators of Energy Use in Urban Systems
}

\author{
Authors: Mark T. Brown, Marco Raugei, Silvio Viglia
}

\section{Introduction}

From 1998 onwards the Biennial International Workshop "Advances in Energy Studies” (BIWAES) has gathered a community of scientists, industry and business energy experts, administrators and policy-makers, social and environmental stakeholders, converging in different locations of the world to present and discuss advances, innovations and visions in energy and energy-related environmental and socioeconomic issues and models. Renowned energy experts and ecologists have discussed the importance of energy in our society and ecosystems and the ways to better analyze and model their complex relationships. This interdisciplinary effort stems from the awareness that scientific and technological achievements, economic processes, art and creativity, policy-making and governance, information networks, even religions and worldwide social movements, may all contribute to different extents to appropriate resource and environmental management and fruition, and promote access to development means, better quality of life and environmental protection. This series of Advances in Energy Studies Workshops aims at sharpening scientific focus and building a critical level of collaborative networking among scientists that deal with energy issues. The work toward this goal has been gaining momentum in recent years, as societal attention once again is shifting toward policy debates concerning the sustainable use of energy and resources and their relationship to the economies of the world.

Previous editions of BIWAES have focused on energy flows in ecology and economy; analysis of the supply side; the ecological consequences of energy sources exploitation; and the role of renewable energy sources and new energy carriers.

The $1^{\text {st }}$ edition "Energy Flows in Ecology and Economy" (1998) was aimed at revitalizing the discussion over theoretical aspects of Energy Analysis, and discussing policy initiatives and future research towards a better balance of humanity and the environment. The $2^{\text {nd }}$ edition "Exploring Supplies, Constraints, and Strategies" (2000) moved beyond a cross-comparison among methods of analysis, and presented original scientific results related to energy supplies, constraints, and strategies, to develop policy and management tools for economy and environment from a systems perspective. The $3^{\text {rd }}$ edition "Reconsidering the importance of energy" (September 2002) questioned the claim that energy analysis is comparatively "unimportant", and focused on energy-related issues within the framework of sustainability, production and consumption patterns, socially acceptable life styles. The $4^{\text {th }}$ edition "Energy and Ecology Issues in Latin America" (June 2004) was dedicated to exploring the uneven access to energy and material resources among countries as well as within countries, due to market mechanisms and unequal trade. The $5^{\text {th }}$ edition "Perspectives on Energy Future" (September 2006) addressed the role of renewable energy sources and new energy carriers, as well as governance issues related to energy policy and the different methods for societal metabolism assessment. The $6^{\text {th }}$ edition "Towards a holistic approach based on science and humanity" (June-July 2008) stressed the importance of energy issues within a perspective of economic and social 
development worldwide, while keeping environmental compatibility in mind. The $7^{\text {th }}$ edition "Can we break the addiction to fossil energy?" (October 2010) analyzed the metabolic patterns of societies, the viability of alternative energy sources and different scenarios of energy transition away from fossil energy. The 8th edition "Energy Security and Development-The Changing Global Context" (October 2012) focused its attention on energy security, taking into account the increasing interdependence of world economies, regardless of the political boundaries that separate them. Finally, we present in this Special Issue a selection of the scientific contributions provided to and discussed during the $9^{\text {th }}$ edition of BIWAES, held in Stockholm, Sweden, 04 - 07 May 2015, and focused on issues of urban metabolism and sustainability.

\section{Sustainable development of urban systems}

Cities are by definition places of convergence and divergence, storage and redistribution: convergence of people, traditions, materials, and energy; divergence of innovation, products, culture, information. Socially and environmentally concerned analysts, planners, managers and policy makers cannot ignore the opportunities that may arise from a sustainable and well-managed urban environment. Concepts such as "sustainable community", "city metabolism", "transition city", "resilient city", "smart city", etc., are gaining the attention of policy makers, managers and stakeholders, and place the focus on the social dimensions of development, as well as on the related ecological and economic aspects and on the innovative technologies and tools that are increasingly becoming available. As societies move away from fossil fuels towards increased use of renewable energy and materials by implementing these "green" technologies and eco-conscious designs, also lifestyles change accordingly, and drive new production and consumption patterns. In this context, a sustainable urban community can be defined as an organization of people, institutions, activities and processes aimed at fulfilling the basic needs of the present and future generations, without disregarding the needs of other species in the same area, within the constraints imposed by the available natural capital and ecosystem services. Perhaps for the first time in the history of our planet, modern transportation and communication technologies are generating an interconnected web of energy, resources, culture and information, in cities and throughout the world, capable to spur awareness and support efforts towards sustainable resource use, social cohesion and equity, inclusion and - ultimately - happiness. However, an opposite outcome may also come to pass if resources and networking opportunities are not properly exploited through proactive attitudes and policies.

The ways societies self- organize, grow, change and decline are affected by and in turn affect resource availability. Understanding the dynamics of resource use and lifestyles in modern cities, how they are affected by resource diversity, environmental problems, land use change and production patterns, allows to shed light on a series of interlinked future trends, among which: energy use, city sizes, sustainability and resilience, interaction with surrounding territories and faraway countries, and networking opportunities with other urban systems for enhancement of urban quality of life.

Quantifying resource use trends is not an easy task, nor something that can be addressed by means of uni-dimensional indicators (e.g., energy price, energy efficiency, energy per person). Instead, it requires multi-dimensional evaluations to simultaneously account for availability, feasibility, desirability and environmental effects. However, rather than a problem, methodological differences may be regarded as a strength: insights derived from different approaches can be combined to increase 
our understanding of the complexity of urban social systems and economies and to generate policies that increase urban sustainability. Unfortunately, though, integrated multi-dimensional assessment methods are not yet at a level of maturity where they may be routinely and reliably used by decision makers. They are still in the early stages of development, with an emphasis on indicators and normative standards that might lead to decision support tools.

This Workshop aimed to take a significant step along the path of such development, by addressing a wide range of available energy options, technologies, energy trends and scenarios for cities; identifying environmental, social and economic constraints to and consequences of energy uses; designing frameworks and urban contexts in relation to energy and resources; highlighting stakeholders and policy-maker roles; and exploring the main sectors, critical steps, and improvement potentials within a balanced interplay of resources and development. Invited Speakers, participant Researchers and a number of junior Scientists presented and discussed their findings on direct and indirect energy use in cities. This ultimately allowed the identification of a common roadmap towards a "sustainable energy city platform", by addressing social, economic, and environmental aspects of city energetics.

\section{Papers hosted in this Special issue}

The papers in this special issue addressed different issues of sustainable development of urban systems:

Agostinho et al. highlighted the potentialities of emergy accounting in contributing to the discussions about the limits of growth for urban productive systems. The methodological approach used in this work contributes to assess urban productive systems through a macro-perspective approach using an input-output model of systems functioning.

Buonocore et al. used Life Cycle Assessment (LCA) to compare the environmental performance of different scenarios for wastewater and sludge disposal in urban wastewater treatment plant (WWT) located in Southern Italy. Five different scenarios, characterized by different degrees of recycling within the plant as well as at larger regional scale, were discussed to explore improvements of the environmental performance of the WWT plant. According to their results, improvements of the wastewater and sludge management could be implemented by adopting additional circular strategies at larger scale, after careful LCA evaluation. Moreover, an improved wastewater treatment plant should not be considered a potential energy source (in spite of the biogas and syngas generation and additional biomass production) but instead a self-sufficient facility providing the much more important water treatment service at low or no energy cost.

Chifari et al. proposed a framework to accommodate various indicators referring to the socioeconomic performance of the Urban Waste Management Systems (viability and desirability) and those related to environmental impact/stress (feasibility). In this paper, such theoretical considerations were illustrated with preliminary data from a case study on the Metropolitan Area of Naples, Italy. 
Corcelli et al. explored solutions for the future urban electricity supply chain. The rapid expansion of the photovoltaic (PV) industry will translate into a large amount of modules to be disposed of in the next years. The Authors evaluated through the LCA different end of life options of photovoltaic panels. In particular, they compared two different end-of-life scenarios, by evaluating the environmental advantages of replacing virgin materials with recovered materials, with a special focus on the steps and/or components of the system that could be further improved. The authors also proved that the recovery process of the PV panels has clear advantages from the energy and environmental points of view in all impact categories analyzed, and at acceptable energy cost.

Dong et al. explored methodologies and technical supports to identify spatial clusters of environmental problems. In particular, their study attempted to explore the spatial patter of Points Of Environmental Problems (POEPs) in the Dongcheng District of Beijing (China) and the relationship with the Points of interest (POIs), to provide suggestions to optimize the allocation of urban management resources and improve management efficiency.

Sevegnani et al. used the Emergy Accounting method to evaluate the internal stocks of the multiurban system called ABC Paulista in Brasil, and the role of stocks in ABC development and growth. Their work led to the estimation of the contribution of storages to produce one unit of GDP, and the potential losses due to stocks changes or overexploitation. Moreover, it may help policy makers to not only address cities' sustainability, but also formulate policies for specific settlements.

Viglia et al proposed a methodology to calculate emergy-based indicators to measure the environmental loading and sustainability of urban systems. In order to implement comprehensive indicators and suggest resource use criteria at urban level, the authors discussed and developed a new metric to estimate the environmental impact and sustainability of five urban systems of different size in Italy, with reference to their resource use environmental sustainability conditions

\section{Conclusions}

A coherent energy strategy is required, to address issues of energy supply and demand in urban areas, also taking into account security of access to energy, a range of development problems, and market dynamics. Energy efficiency and the rebound effect must also be taken into proper account. In the short term, the aim should be to achieve higher energy efficiencies and increased supply from local energy sources, in particular renewable ones. In the long term, a redesign of lifestyles, a further reliance on alternative energy sources and a shift to a more suitable and efficient mix of energy carriers are expected to contribute to solving or at least alleviating the problems generated by the declining availability of fossil fuels. National-level economic accounting procedures are also needed to consider overall resource depletion and environmental degradation, and address questions concerning growth, carrying capacity, sustainability, and inter- and trans-generational equity.

In the end, however, increased efficiency (in buildings, in transportation devices and infrastructure, as well as in production sectors) is still a clear priority, which can be addressed in a number of ways, among which: 
a) Decentralization of energy production, by generating heat and electricity locally by means of small/medium scale conversion plants, in order to make use of locally available resources (biomass, residues, small energy storages) and save on energy transportation infrastructure.

b) Adequate matching of supply to end uses, both in terms of quantity and quality. This especially applies to thermal energy demand of the household sector and small and medium agricultural and industrial enterprises, which can often be easily and safely met by means of solar thermal and biomass devices.

c) So-called "Zero Emission Technologies and Systems", based on clustering of local production systems in order to facilitate the exchange of energy and unused resources, thereby preventing them from becoming waste flows to be disposed of. Achieving "more with less" is a new science- and innovation-based strategy to generate non-linear business cycles, featuring multiple reuse and recycling pathways.

Determining how all these strategies for energy efficiency may be put into practice, and at what cost (including incentives and regulatory interventions), however, still requires multidisciplinary expertise and more open debate, which should no longer be postponed.

\section{Acknowledgement}

We would like to thank all the reviewers for their time and effort. Without their dedication and expertise, this special issue would not have been possible.

\section{References}

Agostinho, F., Sevegnani, F., Almeida, Cecilia M.V.B., Giannetti, B.F., Exploring the potentialities of emergy accounting in studying the limits to growth of urban systems. Ecological Indicators, in this issue. http://dx.doi.org/10.1016/j.ecolind.2016.05.007

Buonocore, E., Mellino, S., De Angelis, G., Liu, G., Ulgiati, S., Life cycle assessment indicators of urban wastewater and sewage sludge treatment. Ecological Indicators, in this issue. http://dx.doi.org/10.1016/j.ecolind.2016.04.047

Chifari, R., Lo Piano, S., Bukkens, S.G.F., Giampietro, M., A holistic framework for the integrated assessment of urban waste management systems. Ecological Indicators, in this issue. http://dx.doi.org/10.1016/j.ecolind.2016.03.006

Corcelli, F., Ripa, M., Leccisi, E., Cigolotti, V., Fiandra, V., Graditi, G., Sannino, L., Tammaro, M., Ulgiati, S., Sustainable urban electricity supply chain - Indicators of material recovery and energy savings from crystalline silicon photovoltaic panels end-of-life. Ecological Indicators, in this issue. http://dx.doi.org/10.1016/j.ecolind.2016.03.028 
Dong, R., Li, S., Zhang, Y., Zhang, N., Wang, T., Tan, X., Fu, X., Analysis of urban environmental problems based on big data from the urban municipal supervision and management information system. Ecological Indicators, in this issue. http://dx.doi.org/10.1016/j.ecolind.2016.09.020

Sevegnani, F., Giannetti, B.F., Almeida, C.M.V.B., Agostinho, F., Brown, M.T., Accounting for internal stocks in assessing the sustainability of urban systems: The case of ABC Paulista. Ecological Indicators, in this issue. http://dx.doi.org/10.1016/j.ecolind.2016.05.029

Viglia, S., Civitillo, D.F., Cacciapuoti, G., Ulgiati, S., Indicators of environmental loading and sustainability of urban systems. An emergy-based environmental footprint. Ecological Indicators, in this issue. http://dx.doi.org/10.1016/j.ecolind.2017.03.060

\section{Guest Editors}

Mark Brown

Center for Environmental Policy, Phelps Lab, University of Florida

Gainesville, FL32611-86350, USA E-mail address: mtb@ufl.edu (M.T. Brown)

Marco Raugei

Faculty of Technology, Design and Environment, Oxford Brookes University Wheatley Campus, Wheatley OX33 1HX United Kingdom E-mail address: marco.raugei@brookes.ac.uk

Silvio Viglia

Center for Environmental Policy, Phelps Lab, University of Florida Gainesville, FL32611-86350, USA E-mail address: silvio.viglia@ufl.edu 


\section{Associate Editors}

Marco Casazza

'Parthenope' University of Naples, Department of Science and Technologies Centro Direzionale, Isola C4, 80143 Napoli, Italy E-mail address: marco.casazza@uniparthenope.it

Hans Schnitzer

StadtLABOR Innovations for urban Quality of Life Ldt.

Griesgasse 40, 8020 Graz, Austria

E-mail address: hans.schnitzer@stadtlaborgraz.at

\section{BIWAES 2015 Chairs}

Olga Kordas

Centre for a Sustainable Built Environment, KTH Royal Institute of Technology,

Teknikringen 34, Stockholm, Sweden E-mail address: olga.kordas@abe.kth.se

Sergio Ulgiati

'Parthenope' University of Naples, Department of Science and Technologies

Centro Direzionale, Isola C4, 80143 Napoli, Italy E-mail address: sergio.ulgiati@uniparthenope.it 